\title{
Lower respiratory tract infection outcomes are predicted better by an age $>80$ years than by CURB-65
}

\section{To the Editors:}

In a recent issue of the European Respiratory Journal, Bont et al. [1] demonstrated that increasing age, previous hospitalisation, heart failure, diabetes, use of oral glucocorticoids, previous use of antibiotics, a diagnosis of pneumonia and an exacerbation of chronic obstructive pulmonary disease were independent predictors of 30-day hospitalisation or death in patients with lower respiratory tract infections (LRTI). They provided a new scoring system using the variables above for the prognostic predictors in the elderly primary-care patients with LRTI [1].

Although some of the predictor variables have been confirmed by other studies, we would like to point out that their results are very important, much more so than previous results.

Age is a well known risk factor for a poor outcome in LRTI. However, most related studies have recommended that an age $\geqslant 65$ yrs presents the greatest risk for a poor outcome of LRTI or community-acquired pneumonia (CAP) [2-4]. It has been recommended by the British Thoracic Society that a simple clinical prediction rule based on the five clinical features of age, confusion, urea, respiratory rate and blood pressure (the CURB65 score) may be a practical means of stratifying patients with CAP into low-, intermediate- and high-mortality risk groups [4]. However, the study by Bont et al. [1] clearly indicated that an age $>80$ yrs presents the greatest risk for a poor outcome of LRTI.

We prospectively examined hospitalised pneumonia patients for 3 yrs (fig. 1). Most of the hospitalised pneumonia patients were $\geqslant 65$ yrs old. In fact, $75 \%$ of hospitalised patients with pneumonia were aged $>70 \mathrm{yrs}$; therefore, an age $\geqslant 65 \mathrm{yrs}$ cannot be a meaningful cut-off level in terms of hospital

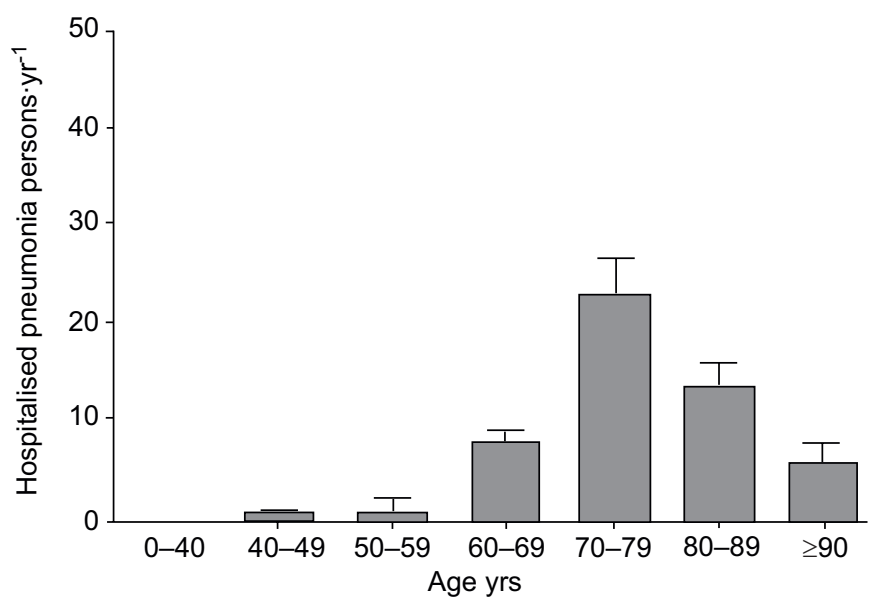

FIGURE 1. The mean \pm SD values of pneumonia incidence in the different ages for 3 yrs are presented admission risk and pneumonia risk. A similar phenomenon may occur in all developed countries, since the aged population is growing very rapidly; thus, in developed countries, CURB-65 may not be advantageous in the prediction of poor outcome in hospitalised LRTI or CAP. Unfortunately, most other studies in this area, which include a low number of elderly subjects, do not examine the significance of new age criteria, such as age $\geqslant 80 \mathrm{yrs}$, being a better predictor for poor outcome than the conventional age criteria determined as age $\geqslant 65 \mathrm{yrs}$.

It has been recently suggested that CURB-65 should not be supplanted by systemic inflammatory response syndrome (SIRS) or the standardised early warning score (SEWS) for initial prognostic assessment in CAP. Further research to identify better generic prognostic tools is required [5]. Although the SIRS and SEWS are different from LRTI and CAP, variables of age and pneumonia may be common contributors for the prognosis.

We would like to reinforce the fact that aspiration and silent aspiration are very important mechanisms of aspiration pneumonia in the elderly [6-9]. Since silent aspirations are very common in patients with stroke and frail elderly patients with advancing age, aspiration risk and dysphagia are significant predictors for the development of pneumonia and poor outcome of LRTI. Suspected aspiration is associated with more aggressive antibiotic treatment of suspected pneumonia episodes in nursing home residents dying with advanced dementia [10]

In conclusion, the current authors respect the fact that the CURB-65 score is useful to predict the outcome of patients with lower respiratory tract infections in the general population. However, the age cut-off point should be seriously reconsidered as significant as a good predictor for the outcome in the current clinical settings in aged populations of developed countries.

S. Teramoto*, H. Yamamoto*, Y. Yamaguchi*, Y. Hanaoka*, M. Ishii*, S. Hibi*, H. Kume ${ }^{\#}$ and Y. Ouchi*

*Depts of Geriatric Medicine, and "Urology, Graduate School of Medicine, University of Tokyo, Tokyo, Japan.

\section{STATEMENT OF INTEREST}

None declared.

\section{REFERENCES}

1 Bont J, Hak E, Hoes AW, Schipper M, Schellevis FG, Verheij TJ. A prediction rule for elderly primary-care patients with lower respiratory tract infections. Eur Respir J 2007; 29: 969-975.

2 Fine MJ, Auble TE, Yealy DM, et al. A prediction rule to identify low-risk patients with community-acquired pneumonia. N Engl J Med 1997; 336: 243-250. 
3 Hak E, Wei F, Nordin J, Mullooly J, Poblete S, Nichol KL. Development and validation of a clinical prediction rule for hospitalization due to pneumonia or influenza or death during influenza epidemics among community-dwelling elderly persons. J Infect Dis 2004; 189: 450-458.

4 Lim WS, van der Eerden MM, Laing R, et al. Defining community acquired pneumonia severity on presentation to hospital: an international derivation and validation study. Thorax 2003; 58: 377-382.

5 Barlow G, Nathwani D, Davey P. The CURB65 pneumonia severity score outperforms generic sepsis and early warning scores in predicting mortality in communityacquired pneumonia. Thorax 2007; 62: 253-259.

6 Teramoto S, Ishii T, Yamamoto $\mathrm{H}$, et al. Significance of chronic cough as a defence mechanism or a symptom in elderly patients with aspiration and aspiration pneumonia. Eur Respir J 2005; 25: 210-211.

7 Teramoto S, Matsuse T, Fukuchi Y, Ouchi Y. Simple twostep swallowing provocation test for elderly patients with aspiration pneumonia. Lancet 1999; 353: 1243.

8 Teramoto S, Ishii T, Yamamoto H, Yamaguchi Y, Ouchi Y. Nasogastric tube feeding is a cause of aspiration pneumonia in ventilated patients. Eur Respir J 2006; 27: 436-437.

9 Teramoto S, Yamamoto H, Yamaguchi Y, et al. ACE inhibitors prevent aspiration pneumonia in Asian, but not Caucasian, elderly patients with stroke. Eur Respir J 2007; 29: 218-219.

10 Chen JH, Lamberg JL, Chen YC, et al. Occurrence and treatment of suspected pneumonia in long-term care residents dying with advanced dementia. J Am Geriatr Soc 2006; 54: 290-295.
From the authors:

We would like to thank S. Teramoto and co-workers for the important issues they raised. While appraising their comments, it is important to make a distinction between the use of severity rules inside and outside hospital settings. Looking at the available literature, we think that the pneumonia severity index (PSI) and CURB-65 (Confusion, Urea $>7 \mathrm{mmol} \cdot \mathrm{L}^{-1}$, Respiratory rate $\geqslant 30$ breaths $\cdot \mathrm{min}^{-1}$, Blood pressure (systolic value $<90 \mathrm{mmHg}$ or diastolic value $\leqslant 60 \mathrm{mmHg}$ ) ) are both valid and useful in hospital settings. However, it is an interesting suggestion to improve CURB-65 by introducing more detailed age groups in the score. In primary care, PSI and CURB-65 are less useful for various reasons. Regarding the predictive value of age, the results of our study [1] showed that age $>80$ yrs was a better predictor of outcome than age categories between 65 80 yrs. Probably as there are a lot of healthy individuals aged 65-80 yrs in primary care who have a low risk for poor outcome.

\section{T. Verheij, J. Bont, E. Hak and A. Hoes}

University Medical Center Utrecht, Utrecht, The Netherlands.

\section{STATEMENT OF INTEREST}

None declared.

\section{REFERENCES}

1 Bont J, Hak E, Hoes AW, Schipper M, Schellevis FG, Verheij TJ. A prediction rule for elderly primary-care patients with lower respiratory tract infections. Eur Respir J 2007; 29: 969-975.

\section{Oral antibiotics prior to hospitalisation for community- acquired pneumonia}

\section{To the Editor:}

SCHAAF et al. [1] postulate that antibiotics prior to hospitalisation with community-acquired pneumonia may be protective because of a slightly lower death rate and lower C-reactive protein concentration, leukocyte count and acute physiology score in the 13 out of 105 patients that received them. Since AUSTRIAN and GOLD [2] demonstrated a reduction in mortality from 80 to $17 \%$ in bacteraemic pneumococcal infections treated with penicillin, the death rate for this condition has changed little. A 2006 study has suggested that deaths in patients with community-acquired pneumonia are far more likely to be due to host factors rather than antibiotic choices [3].

It is possible that such host factors could lead to some patients having better outcomes, subacute presentations and more time before hospitalisation in which to receive oral antibiotics. Conversely, those patients with worse outcomes may show more acute presentations, removing the option of pre-hospitalisation antibiotics. Information on the number of days that patients were unwell prior to admission may help to answer this in part. Given the inaccuracy with which doctors make the diagnosis of community-acquired pneumonia, this is an important point [4-6], since pharmaceutical companies might be predicted to use potentially misleading conclusions such as this to encourage primary care physicians to prescribe antibiotics to anyone who might have community-acquired pneumonia, with potential for increased levels of antibiotic resistance, unnecessary costs and potential side-effects.

\section{P.G.P. Charles}

Dept of Infectious Diseases, Austin Health, Heidelberg, Australia.

\section{STATEMENT OF INTEREST}

None declared. 